\title{
Clinical significance of monocyte-derived dendritic cell activation in patients with acute coronary syndrome
}

\author{
Yasue Takahashi ${ }^{1}$, Kazunori Shimada ${ }^{1}$, Katsuhiko Sumiyoshi ${ }^{1}$, Takashi Kiyanagi ${ }^{1}$, Makoto Hiki ${ }^{1}$, \\ Kosuke Fukao $^{1}$, Kuniaki Hirose ${ }^{1}$, Rie Matsumori ${ }^{1}$, Hiromichi Ohsaka ${ }^{1}$, Atsumi Kume ${ }^{1}$, \\ Tetsuro Miyazaki ${ }^{1}$, Hiroaki Miyajima ${ }^{2}$, Isao Nagaoka ${ }^{3}$, Hiroyuki Daida ${ }^{1}$
}

\author{
${ }^{1}$ Department of Cardiovascular Medicine, Juntendo University School of Medicine, Tokyo, Japan \\ ${ }^{2}$ Division of Cell Biology, Juntendo University Graduate School of Medicine, Tokyo, Japan \\ ${ }^{3}$ Department of Host Defense and Biochemical Research, Juntendo University Graduate School of Medicine, Tokyo, Japan \\ Email:shimakaz@juntendo.ac.jp
}

Received 13 December 2011; revised 10 January 2012; accepted 21 January 2012

\section{ABSTRACT}

Background: Acute coronary syndrome (ACS) is an amplified state of inflammation and immune reaction. Dendritic cells (DCs) expressing various Toll-like receptors (TLRs) have been observed in atherosclerotic lesions, however, the clinical significance of DCs in pathogenesis of ACS has not been completely investigated. Methods: Ten patients with ACS and 10 patients with stable angina pectoris (SAP) were enrolled in this study. Monocyte-derived DCs were generated from $\mathrm{CD}^{+} 4^{+}$cells by culturing with granulocyte macrophage colony-stimulating factor and interleukin (IL)-4 for 6 days. Expression of cell surface CD86 and CD83 were measured by flowcytometry. Expression of genes, including CD86, CD83, CCL19, CCR7, TLR2, TLR4, TLR5, TLR8, and TLR9, were measured by real-time PCR. Plasma IL-6 and tumor necrosis factor (TNF)- $\alpha$ levels were also measured. Results: The number of $\mathrm{CD86}^{+} \mathrm{CD83}^{+} \mathrm{DCs}$ in the ACS group was significantly higher than that in the SAP group $(P<0.05)$. Expression levels of CD86, CD83, CCL19, CCR7, TLR2, TLR4, and TLR9 in the ACS group were significantly higher than those in the SAP group (all, $P<0.05$ ). Plasma IL-6 and TNF- $\alpha$ levels in the ACS group were significantly higher than those in the SAP group (all, $P<0.05$ ). In addition, a positive correlation was observed between the number of $\mathrm{CD86}^{+} \mathrm{CD83}^{+}$cells and plasma levels of IL-6 $(r=0.88$, $P<0.0001)$ as well as between the number of CD86 $^{+} \mathrm{CD83}^{+}$cells TNF- $\alpha$ levels $(r=0.78, P<0.0001)$. Conclusions: These results demonstrated that monocyte-derived DCs are activated in patients with ACS, suggesting that activated DCs may play an important role in the pathogenesis of ACS.

Keywords: Acute Coronary Syndrome (ACS); Dendritic
Cells (DCs); Toll-Like Receptors (TLRs)

\section{INTRODUCTION}

Inflammation is an important factor in the initiation and development of atherosclerosis [1-4]. Various types of inflammatory cells, such as monocytes, macrophages, dendritic cells (DCs), and lymphocytes, participate in the processes [5]. Furthermore, all these cells are also immunereactive cells. Innate immunity and adaptive immunity have been implicated in all stages of atherosclerosis, from initiation through progression and in atherothrombotic complications $[3,5]$. DCs as professional antigen-presenting cells (APCs) utilize pathogen-associated molecular patterns receptors including scavenger recaptors and Toll-like receptors (TLRs) on the cell surface [69]. Recent studies have demonstrated the relationship among circulating myeloid DCs, plasmacytoid DCs, and coronary artery disease (CAD) including stable angina pectoris (SAP) and acute coronary syndrome (ACS). However, the results of each study are not entirely in agreement [10-13].

A broad spectrum of TLRs, including TLR1, TLR2, TLR4, and TLR5, are found in atherosclerotic plaques [14]. Expression of TLR4 on circulating monocytes in patients with SAP and unstable angina (UAP) is higher than that in controls [15]. While these data indicate that TLRs may play an important role in the pathophysiological relationship between innate immunity and atherosclerosis, few studies have investigated the association between expression of TLRs on DCs and cardiovascular events.

In this study, we generated monocyte-derived DCs from patients with ACS and those with SAP by culturing with granulocyte macrophage colony-stimulating factor (GM-CSF) and interleukin (IL)-4. We assessed activating markers on the cell surface, gene expressions of 
various TLRs, and DC-associated chemokine in monocyte-derived DCs in each group. In addition, circulating levels of inflammatory cytokines were measured and compared among groups.

\section{METHODS}

\subsection{Subjects}

Ten consecutive patients with ACS [3 with acute myocardial infarction (AMI) and 7 with UAP] who underwent emergency coronary angiography and 10 patients with SAP who underwent diagnostic coronary angiography at the same time at the Juntendo University Hospital were enrolled in this study. All patients had documented CAD defined as more than $50 \%$ stenosis in at least one major coronary artery. AMI was defined as the presence of typical prolonged chest pain associated with significant elevation of myocardial enzymes and the appearance of abnormal $\mathrm{Q}$ waves and/or $\mathrm{T}$ wave inversion on the standard 12-lead electrocardiogram. SAP was defined as stable effort angina and electrocardiographic changes $\geq 0.1 \mathrm{mV}$ ST depression during the exercise stress test with normal left ventriculograms and without history of ACS. Patients with AMI were referred within $6 \mathrm{~h}$ of onset and all patients with UAP were classified as class III. Patients with ongoing congestive heart failure and those who had liver and/or renal dysfunction were excluded. Written informed consent was obtained from all subjects and the ethics committee of the institution approved this study.

\subsection{Blood Sampling and Cell Culture}

Blood samples were obtained from patients immediately before coronary angiography. Monocytes were purified from peripheral blood mononuclear cells of subjects by magnetic bead separation (Miltenyi Biotec). To generate DCs, purified CD14 ${ }^{+}$cells were cultured in a medium with $800 \mathrm{U} / \mathrm{mL}$ GM-CSF (R\&D Systems) and 1000 $\mathrm{U} / \mathrm{mL}$ IL-4 (R\&D Systems) for 6 days, as previously described [16]. These DCs displayed typical DC morphology and were confirmed negative for CD14 and highly positive for HLA-DR.

\subsection{Flowcytometry}

Expression of CD83 and CD86 on the surface of DCs was analyzed with CD86-fluorescein isothiocyanate and CD83-PE, respectively. Cell fluorescence intensities were compared with isotype-matched control antibodies. Ten thousand events were measured by flow cytometry (FACScan, Becton Dickinson), and data were analyzed with WinMDI software.

\subsection{Real-Time PCR}

Total RNA from monocyte-derived DCs was extracted using the RNeasy Mini Kit (Qiagen) and treated with RNase-free DNase according to the manufacturer's instructions. Next, $1 \mu \mathrm{g}$ RNA was reverse transcribed to cDNA using the High Capacity cDNA Reverse Transcription Kit (Applied Biosystems, Foster City, CA, USA). Real-time PCR (7500 Real-time PCR System, Applied Biosystems) was used to determine mRNA levels of CD86, CD83, CCL19, CCR7, TLR2, TLR4, TLR5, TLR8, and TLR9, as described previously [17]. TaqMan ${ }^{\circledR}$ Gene Expression Assays for CD83

(Cat\# Hs00188486_m1), CD86 (Cat\# Hs01567026_m1), CCL19 (Cat\# Hs00171149_m1), CCR7

(Cat\# Hs01013469_m1), TLR2 (Cat\# Hs01014511_m1), TLR4 (Cat\# Hs00152939 m1), TLR5

(Cat\# Hs00152825_m1), TLR8 (Cat\# Hs00607866_mH), TLR9 (Cat\# Hs00370913_s1), and $\beta$-actin

(Cat\# Hs99999903_m1) were purchased from Applied Biosystems. The data were normalized relative to $\beta$-actin as an internal control, as described previously [17].

\subsection{Biochemical Analysis}

White blood cell count and serum total cholesterol, triglyceride, high-density lipoprotein (HDL)-cholesterol, and high sensitivity C-reactive protein (hs-CRP) levels were determined by standard methods. Low-density lipoprotein-cholesterol levels were calculated by the Friedewald's formula. HbA1c levels were estimated as $\mathrm{Na}$ tional Glycohemoglobin Standardization Program equivalent value (\%) were estimated using the formula: HbAlc $(\%)=$ HbA1c [Japan Diabetic Society (JDS)] (\%) $+0.4 \%$, considering the relational expression of $\mathrm{HbA} 1 \mathrm{c}$ (JDS) (\%) as measured by the previous Japanese standard substance and measurement methods [18]. IL-6 and TNF- $\alpha$ levels were measured by an enzyme-linked immunosorbent assay (R\&D Systems).

\subsection{Statistical Analysis}

Statistical intergroup differences were analyzed by the chi-square test and the Student's $t$ test. Correlation between the 2 parameters was determined by simple linear regression analysis. $P<0.05$ was considered statistically significant.

\section{RESULTS}

\subsection{Characteristics of Study Subjects}

The characteristics of the subjects are shown in Table 1. There were no significant differences in age, gender, body mass index, lipid profiles, hs-CRP levels, prevalence of hypertension, diabetes, or smoking history between the two groups.

\subsection{Activating Markers on the Surface of Monocyte-Derived DCs}

Figure 1(a) shows representative data of surface markers 
Table 1. Characteristics of study subjects.

\begin{tabular}{lccc}
\hline & SAP & ACS & $\boldsymbol{P}$ \\
\hline Number & 10 & 10 & \\
Age, yrs & $64 \pm 15$ & $62 \pm 13$ & 0.722 \\
Male (\%) & $8(80)$ & $9(90)$ & 0.556 \\
Body mass index, kg/m² & $23.3 \pm 3.3$ & $21.9 \pm 3.0$ & 0.323 \\
Hypertension (\%) & $5(50)$ & $6(60)$ & 0.673 \\
Diabetes (\%) & $3(30)$ & $3(30)$ & 0.999 \\
Smokers (\%) & $4(40)$ & $7(70)$ & 0.279 \\
Family history (\%) & $3(33)$ & $2(20)$ & 0.537 \\
Total cholesterol, mg/dL & $207 \pm 28$ & $191 \pm 43$ & 0.341 \\
Triglyceride, mg/dL & $107 \pm 33$ & $115 \pm 62$ & 0.726 \\
HDL-cholesterol, mg/dL & $54 \pm 18$ & $50 \pm 23$ & 0.674 \\
LDL-cholesterol, mg/dL & $126 \pm 35$ & $123 \pm 37$ & 0.854 \\
White blood cels, 10 ${ }^{3} \mathrm{uL}$ & $5.7 \pm 1.8$ & $7.6 \pm 2.4$ & 0.062 \\
Red blood cells, 10 ${ }^{4} \mathrm{uL}$ & $450 \pm 60$ & $455 \pm 113$ & 0.907 \\
& $6.0 \pm 1.3$ & $6.1 \pm 0.8$ & 0.757 \\
HbA1c, \% (JDS) & $(5.6 \pm 1.2)$ & $(5.7 \pm 0.7)$ & \\
& $0.051 \pm 0.061$ & $0.978 \pm 2.25$ & 0.210 \\
\hline hsCRP, mg/L & & & \\
\hline
\end{tabular}

of monocyte-derived DCs measured by flowcytometry in a patient with ACS and a patient with SAP. The prevalence of $\mathrm{CD} 86^{+}, \mathrm{CD} 83^{+}$, and $\mathrm{CD} 83^{+} \mathrm{CD} 86^{+}$on monocytederived DCs in the patient with ACS was higher than that in the patient with SAP. Expression levels of $\mathrm{CD}^{+} 6^{+}$ $(57 \% \pm 19 \%$ vs. $37 \% \pm 14 \%, P<0.05), \mathrm{CD}^{+} 3^{+}(25 \% \pm$ $13 \%$ vs. $17 \% \pm 11 \%, P<0.05)$, and $\mathrm{CD}^{2} 3^{+} \mathrm{CD}^{+} 6^{+}(20 \%$ $\pm 9 \%$ vs. $13 \% \pm 6 \%, P<0.05)$ on monocyte-derived DCs in the ACS group were significantly higher than those in the SAP group (Figure 1(b)). There was no significant difference in expression of levels of $\mathrm{CD}^{+} 6^{+}$, $\mathrm{CD}^{+} 3^{+}$, or $\mathrm{CD}_{3}{ }^{+} \mathrm{CD} 86^{+}$between the AMI and the UAP groups.

\section{3. mRNA Expression of Activating Markers and TLRs in Monocyte-Derived DCs}

Figure 2 shows the mRNA expression levels of activating and inflammatory markers in monocyte-derived DCs. Expression levels of CD86 (1.97 \pm 0.55 vs. $1.20 \pm 0.41$, $P<0.005), \mathrm{CD} 83$ (4.47 \pm 3.62 vs. $1.82 \pm 0.65, P<0.05)$, CCL19 (2.15 \pm 1.04 vs. $1.19 \pm 0.38, P<0.05)$, and CCR7 $(5.09 \pm 2.30$ vs. $2.17 \pm 0.71, P<0.005)$ in the ACS group were significantly higher than those in the SAP group.

Figure 3 shows the mRNA expression levels of TLRs in monocyte-derived DCs. Expressions levels of TLR2 $(4.15 \pm 3.91$ vs. $1.25 \pm 0.67, P<0.005)$, TLR4 $(2.08 \pm$ 0.54 vs. $1.18 \pm 0.50, P<0.005)$, and TLR9 ( $4.62 \pm 2.83$ vs. $1.55 \pm 0.97, P<0.005)$ in the ACS group were significantly higher than those in the SAP group. No sig- nificant difference in expression of TLR5 or TLR8 was observed.

\subsection{Plasma IL-6 and TNF- $\alpha$ Levels in each Group and the Correlation between the Number of $\mathrm{CD86}^{+} \mathrm{CD83}^{+}$and Cytokine Levels}

Figure 4 shows IL-6 and TNF- $\alpha$ levels in each group. Plasma IL-6 ((5.89 \pm 3.98$)$ vs. $(2.65 \pm 1.95) \mathrm{pg} / \mathrm{mL}, P<$ $0.05)$ and TNF- $\alpha((3.07 \pm 2.89)$ vs. $(0.42 \pm 0.77) \mathrm{pg} / \mathrm{mL}$, $P<0.05)$ levels in the ACS group were significantly higher than those in the SAP group. In addition, a positive correlation was observed between the number of $\mathrm{CD} 6^{+} \mathrm{CD}^{+} 3^{+}$cells and plasma IL-6 levels $(r=0.88, P<$ $0.0001)$ as well as between the number of $\mathrm{CD} 86^{+} \mathrm{CD} 83^{+}$ cells and TNF- $\alpha$ levels $(r=0.78, P<0.0001)$.

\section{DISCUSSION}

This study demonstrated that patients with ACS had 1) higher number of $\left.\mathrm{CD} 86^{+} \mathrm{CD} 83^{+} \mathrm{DCs}, 2\right)$ higher expression of CD86, CD83, CCL19, CCR7, TLR2, TLR4, and TLR9, 3) higher plasma IL-6 and TNF- $\alpha$ levels in comparison to SAP patients, and 4) a positive correlation between the number of $\mathrm{CD} 6^{+} \mathrm{CD}^{+} 3^{+}$cells and plasma IL-6 and TNF- $\alpha$ levels, suggesting that monocyte-derived DCs are activated in patients with ACS, and activated DCs may play an important role in pathogenesis of ACS.

DCs act as professional APCs that are central to the induction and regulation of adaptive immune responses $[19,20]$. While macrophages act as APCs, DCs have specialized capacities for homing to $\mathrm{T}$ cell zones of lymphoid organs to interact with $\mathrm{T}$ lymphocytes [19,20]. Indeed, activating DCs were observed in atherosclerotic lesions [21-23]. Increased number of DCs was observed in the intimal neovascularization area as well as media and adventitia of vessels with inflamed atherosclerotic lesions [24]. In addition, a recent study using a novel bioengineered human artery model clearly demonstrated that activated DCs, rather than macrophages, induced a braking of $\mathrm{T}$ cell tolerance and initiation of vascular inflammation [16]. Therefore, much attention has been focused on the role of DCs in the initiation and progression of atherosclerosis.

Previous studies reported that the expression of CD80 and CD86 on monocyte-derived DCs in ACS patients was significantly higher than that in SAP patients [24,25]. Our results agree with these previous findings. In addition, the present study confirmed mRNA levels and expression of cell surface molecules, such as CD86 and CD83 in monocyte-derived DCs. Several recent studies reported the correlation among circulating myeloid DCs, plasmacytoid DCs, and CAD, however, the results of 

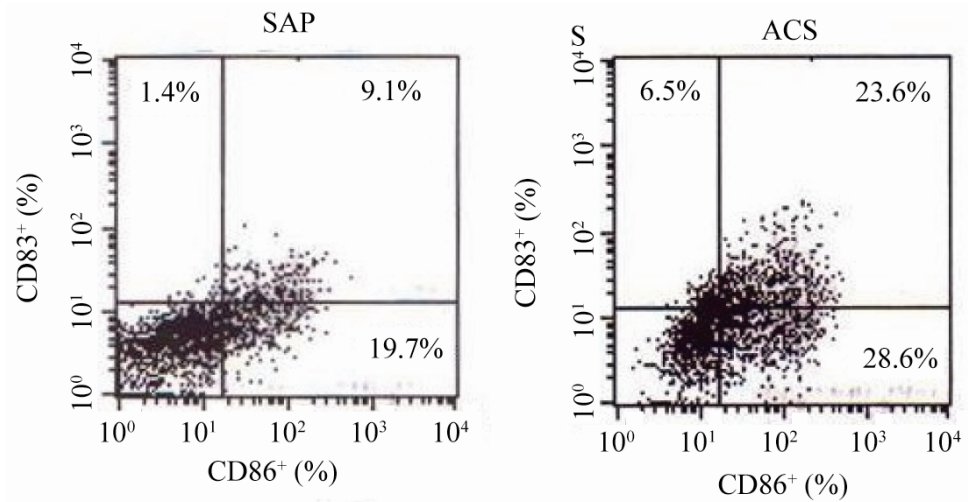

(a)
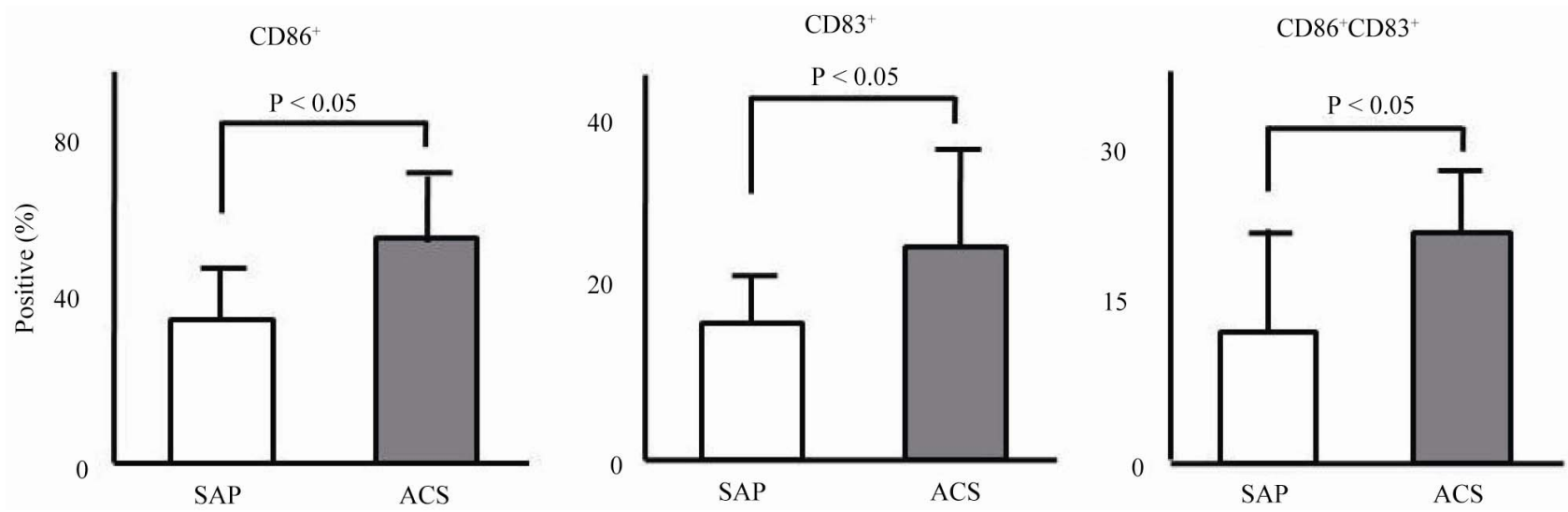

(b)

ACS: acute coronary syndrome, SAP: stable angina pectoris.

Figure 1. Activating markers on the surface of monocyte-derived DCs. (a) Representative data of surface markers of monocyte-derived DCs measured by flowcytometry in a patient with ACS and a patient with SAP; (b) Prevalence of CD86 ${ }^{+}, \mathrm{CD}^{+} 3^{+}$, and $\mathrm{CD}^{+} 3^{+} \mathrm{CD} 86^{+}$on monocyte-derived DCs in the ACS and SAP groups.

each study were not necessarily in agreement [10-13]. One possible reason for the discrepancies may be the differences in specific markers used to define each DC subset. In addition, circulating myeloid and plasmacytoid DCs are characterized as precursors [10]. On the other hand, monocyte-derived DCs cultured with GM-CSF and IL-4 in the present study have typical DC morphology and are negative for CD14 and highly positive for HLADR, as described previously [16].

In the present study, we showed that the expression levels of CCL19 and CCR7 in monocyte-derived DCs in the ACS group were significantly higher than those in the SAP group. After acquiring and processing antigens, DCs migrate from peripheral tissues to lymph nodes [26, 27]. This migration depends on CCR7 and its ligand CCL19. CCL19 and CCR7 also play a pivotal role in lymphocyte trafficking by promoting infiltration of $\mathrm{T}$ cells and DCs into lymphoid tissues [26]. A previous study showed a possible pathogenic role of CCL19 and CCR7 in plaque destabilization [26]. In contrast, other studies indicated a possible role of CCR7 in atheroscle- rosis regression [27,28]. Further investigations are required to clarify the role of CCL19 and CCR7 in the initiation and progression of atherosclerosis and in the pathogenesis of ACS.

Innate immunity is the first line of defense that is quickly mobilized to exogenous and endogenous foreign bodies utilizing receptors, such as scavenger receptors and TLRs [6,7]. To date, 11 TLRs and numerous exogenous and endogenous ligands has been identified in mammals $[6,9]$. TLRs have been shown to play a pivotal role in inducing the immune activation in DCs [29]. TLRs recognize pathogen-associated-molecular-patterns, including peptidoglycan, lipoteichoic acid, and lipopolysaccharide, and signal to DCs. DCs maturation induce innate- and acquired-immune systems. Resting DCs or DCs receiving immune-inhibitory signals induce immune tolerance via $\mathrm{T}$ cell deletion and induction of regulatory T cells [29]. A wide spectrum of TLRs, including TLR1, TLR2, TLR4, and TLR5, are found in atherosclerotic lesions [14]. Interestingly, arterial DCs also express TLR1, TLR2, TLR3, TLR4, TLR7, and TLR9 [30,31]. 

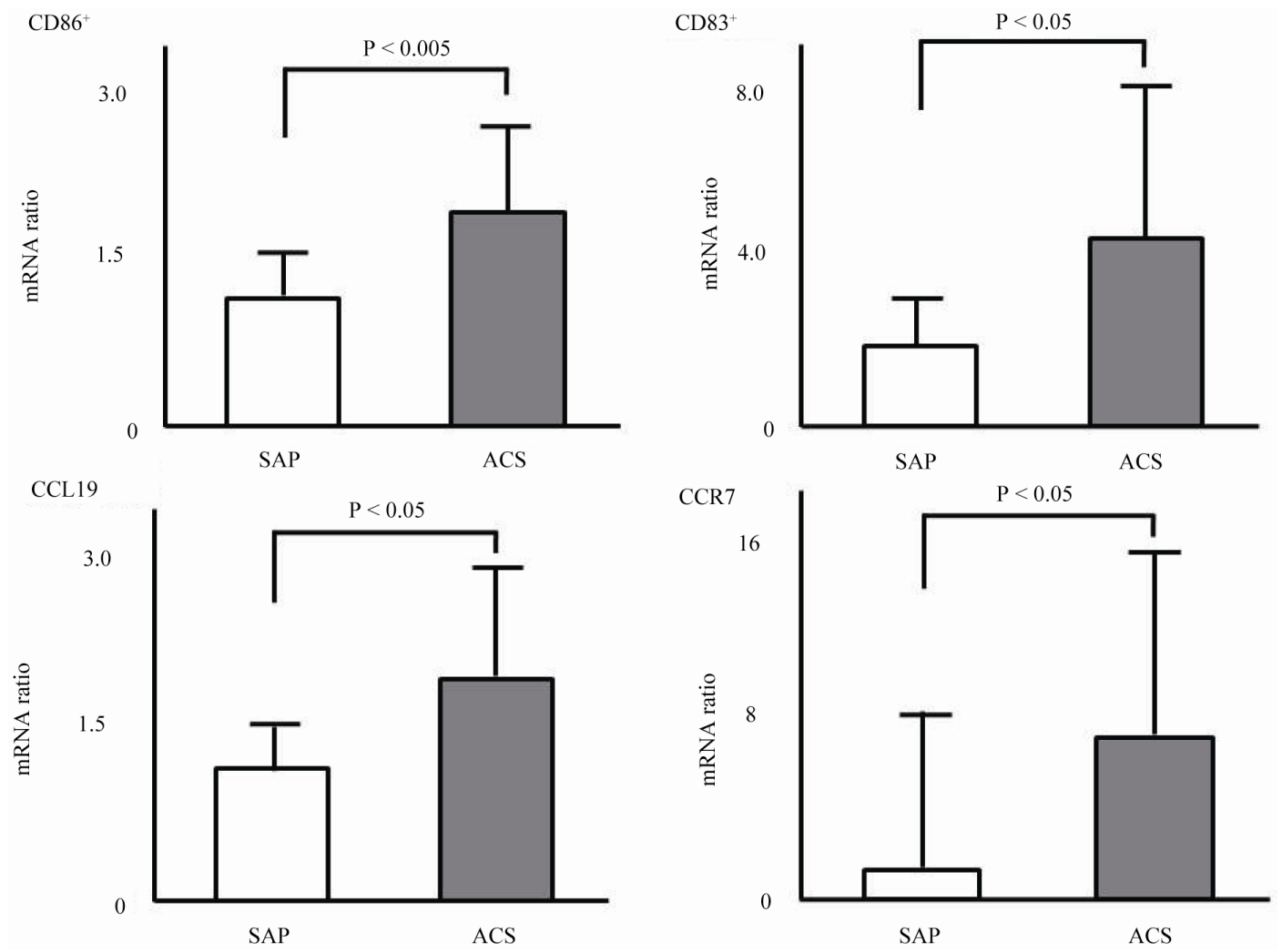

ACS: acute coronary syndrome, SAP: stable angina pectoris, TLR: Toll-like recptor.

Figure 2. mRNA expression of TLRs in monocyte-derived DCs.
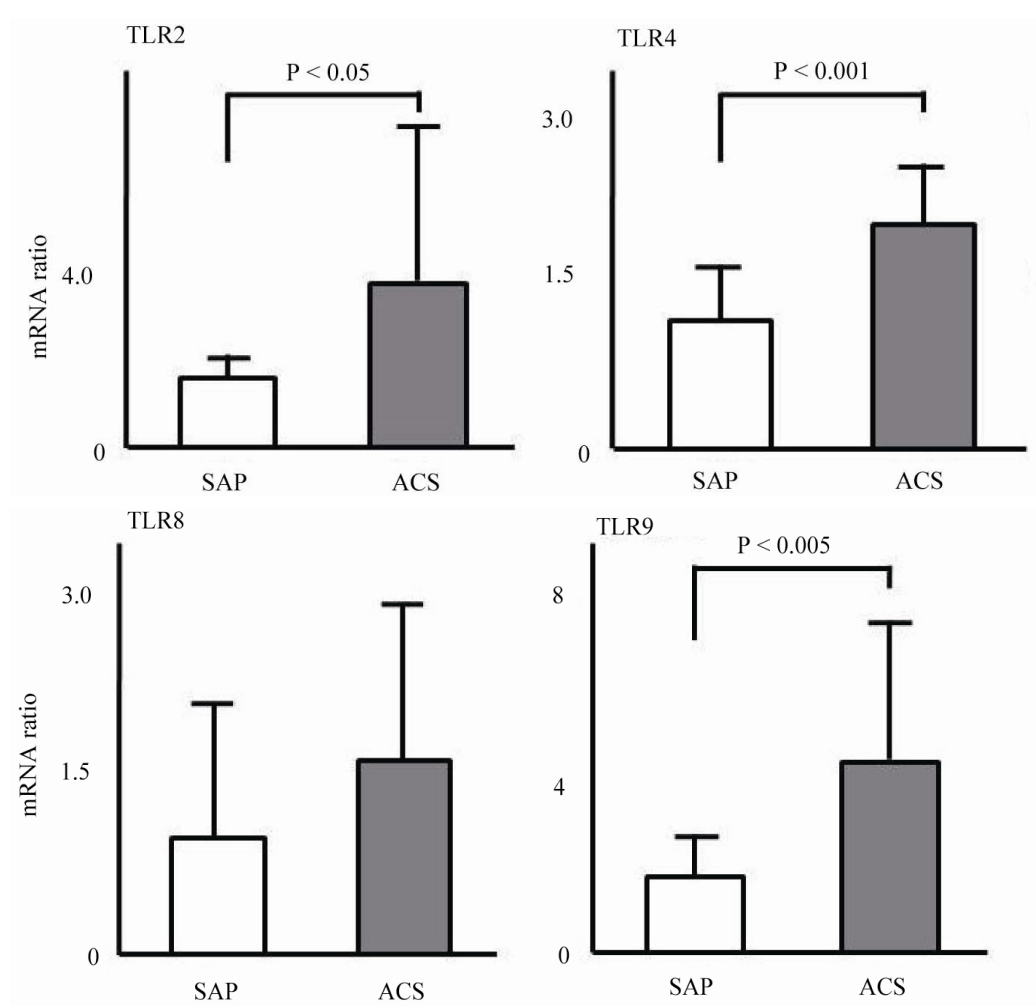

ACS: acute coronary syndrome, SAP: stable angina pectoris.

Figure 3. mRNA expression of activating markers in monocyte-derived DCs. 

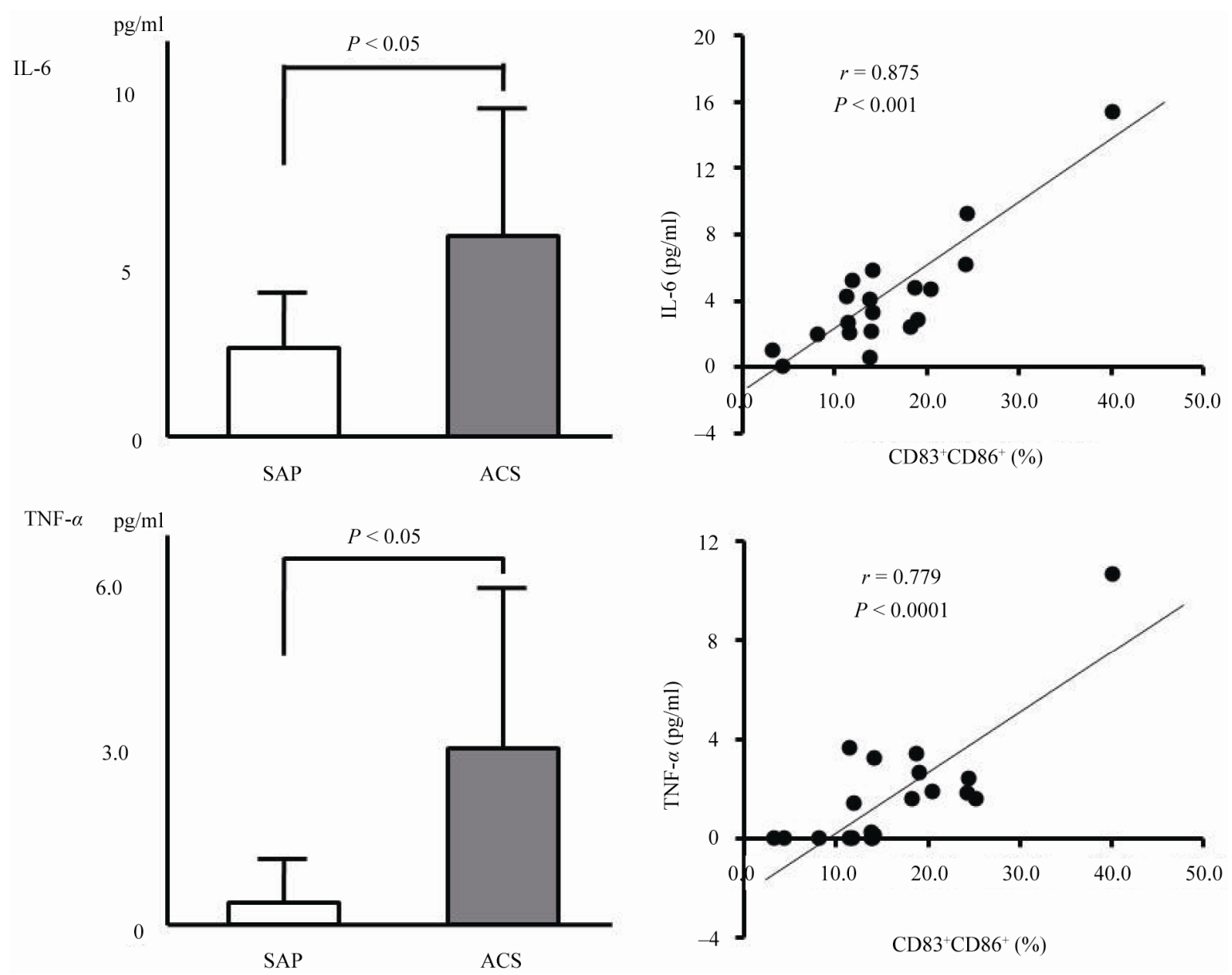

ACS: acute coronary syndrome, SAP: stable angina pectoris, IL: interleukin, TNF: tumor necrosis factor.

Figure 4. Plasma levels IL-6 and TNF- $\alpha$ levels in each group and correlation between the number of CD $86^{+} \mathrm{CD} 83^{+}$cells and cytokine levels.

However, previous studies have mainly showed the expression of TLR4 on circulating monocytes in patients with ACS $[15,32]$. There are few studies that have investigated the association between the expression of TLRs on a broad spectrum of DCs and cardiovascular events. In the present study, expression levels of TLR2, TLR4, and TLR9, but not TLR5 and TLR8, in the ACS group were significantly higher than those in the SAP group. TLR2 and TLR4 are ubiquitously present in six different human vessels (aorta, subclavian, carotid, mesenteric, iliac, and temporal arteries), TLR7 and TLR9 are infrequent, and TLR1, TLR3, TLR5, and TLR8 are selectively expressed, suggesting that territorial distribution of TLRs may contribute to tissue tropism for selective vascular lesions [30]. Specific expression of TLRs in DCs may also contribute to the onset of ACS. Further studies are needed to examine this hypothesis.

There are several limitations to the current study. First, our study had a small sample size. However, this preliminary data, to the best of our knowledge, is the first to demonstrate differences in expression levels of CD86,
CD83, CCL19, CCR7, and various TLRs simultaneously in monocyte-derived DCs between the ACS and SAP groups. Second, we were unable to demonstrate a direct association between activation of DCs and ACS onset because of the nature of the cross-sectional study. Therefore, prospective studies with larger sample sizes are required to confirm these results. Third, there were no control subjects without CAD. However, previous studies reported that activating surface markers and expression of TLRs as well as the circulating number of myeloid DCs were not different between the SAP and control groups $[13,25]$.

In conclusion, we showed that monocyte-derived DCs are activated in patients with ACS, suggesting that activated DCs may play an important role in the pathogenesis of ACS. Additional large sample-sized studies are needed to confirm these findings.

\section{REFERENCES}

[1] Ross, R. (1999) Atherosclerosis-An inflammatory dis- 
ease. The New England Journal of Medicine, 340, 115126.

[2] Libby, P. (2002) Inflammation in atherosclerosis. Nature, 420, 868-874.

[3] Hansson, G.K. and Libby, P. (2006) The immune response in atherosclerosis: A double-edged sword. Nature Reviews Immunology, 6, 508-519.

[4] Yamashita, H., Shimada, K., Seki, E., et al. (2003) Concentrations of interleukins, interferon, and C-reactive protein in stable and unstable angina pectoris. American Journal of Cardiology, 91, 133-136. doi:10.1016/S0002-9149(02)03097-7

[5] Shimada, K. (2009) Immune system and atherosclerotic disease: heterogeneity of leukocyte subsets participating in the pathogenesis of atherosclerosis. Circulation Journal, 73, 994-100. doi:10.1253/circj.CJ-09-0277

[6] Akira, S. and Takeda, K. (2004) Toll-Like receptor signalling. Nature Reviews Immunology, 4, 499-511.

[7] Beutler, B. (2004) Inferences, questions and possibilities in Toll-like receptor signaling. Nature, 430, 257-263.

[8] De Kleijn, D. and Pasterkamp, G. (2003) Toll-Like recaptors in cardiovascular diseases. Cardiovascular Research, 60, 58-67. doi:10.1016/S0008-6363(03)00348-1

[9] Shimada, K., Daida, H., Ma-Krupa, W., et al. (2005) Lipopolysaccharide, CD14 and Toll-like receptors: An emerging link between innate immunity and atherosclerotic disease. Future Cardiology, 1, 657-674. doi:10.2217/14796678.1.5.657

[10] Yilmaz, A., Weber, J., Cicha, I., et al. (2006) Decrease in circulating myeloid dendritic cell precursors in coronary artery disease. Journal of the American College of Cardiology, 48, 70-80. doi:10.1016/j.jacc.2006.01.078

[11] Van Vré, E.A., Hoymans, V.Y., Bult, H., et al. (2006) Decreased number of circulating plasmacytoid dendritic cells in patients with atherosclerotic coronary artery disease. Coronary Artery Disease, 17, 243-248. doi:10.1097/00019501-200605000-00007

[12] Shi, H., Ge, J., Fang, W., et al. (2007) Peripheral-Blood dendritic cells in men with coronary heart disease. American Journal of Cardiology, 100, 593-597. doi:10.1016/j.amjcard.2007.03.067

[13] Fukunaga, T., Soejima, H., Irie, A., et al. (2009) High ratio of myeloid dendritic cells to plasmacytoid dendritic cells in blood of patients with acute coronary syndrome. Circulation Journal, 73, 1914-1919. doi:10.1253/circj.CJ-08-1193

[14] Edfeldt, K., Swedenborg, J., Hansson, G.K., et al. (2002) Expression of toll-like receptors in human atherosclerotic lesions: A possible pathway for plaque activation. Circulation, 105, 1158-1161.

[15] Vink, A., Schoneveld, A.H., van Der Meer, J.J., et al. (2002) In vivo evidence for a role of toll-like receptor 4 in the development of intimal lesions. Circulation, 106, 1985-1990. doi:10.1161/01.CIR.0000032146.75113.EE

[16] Han, J.W., Shimada, K., Ma-Krupa, W., et al. (2008) Vessel wall-embedded dendritic cells induce T-cell autoreactivity and initiate vascular inflammation. Circulation Research, 102, 546-553.

\section{doi:10.1161/CIRCRESAHA.107.161653}

[17] Fukao, K., Shimada, K., Naito, H., et al. (2010) Voluntary exercise ameliorates the progression of atherosclerotic lesion formation via anti-inflammatory effects in apolipoprotein E-deficient mice. Journal of Atherosclerosis and Thrombosis, 17, 1226-1236. doi:10.5551/jat.4788

[18] Seino, Y., Nanjo, K., Tajima, N., et al. (2010) Report of the committee on the classification and diagnostic criteria of diabetes mellitus. Diabetology International, 1, 220. doi:10.1007/s13340-010-0006-7

[19] Shortman, K. and Naik, S.H. (2007) Steady-State and inflammatory dendritic-cell development. Nature Reviews Immunology, 7, 19-30.

[20] Randolph, G.J., Ochando, J. and Partida-Sánchez, S. (2008) Migration of dendritic cell subsets and their precursors. Annual Review of Immunology, 26, 293-316. doi:10.1146/annurev.immunol.26.021607.090254

[21] Bobryshev, Y.V. and Lord, R.S. (1995) S-100 positive cells in human arterial intima and in atherosclerotic lesions. Cardiovascular Research, 29, 689-696.

[22] Bobryshev, Y.V. and Lord, R.S. (1998) Mapping of vascular dendritic cells in atherosclerotic arteries suggests their involvement in local immune-inflammatory reactions. Cardiovascular Research, 37, 799-810. doi:10.1016/S0008-6363(97)00229-0

[23] Yilmaz, A., Lochno, M., Traeg, F., et al. (2004) Emergence of dendritic cells in rupture-prone regions of vulnerable carotid plaques. Atherosclerosis, 176, 101-110. doi:10.1016/j.atherosclerosis.2004.04.027

[24] Ranjit, S., Dazhu, L., Qiutang, Z., et al. (2004) Differentiation of dendritic cells in monocyte cultures isolated from patients with unstable angina. International Journal of Cardiology, 97, 551-555. doi:10.1016/j.ijcard.2004.05.022

[25] Wang, L., Li, D., Yang, K., et al. (2008) Toll-Like receptor-4 and mitogen-activated protein kinase signal system are involved in activation of dendritic cells in patients with acute coronary syndrome. Immunology, 125, 122-130. doi:10.1111/j.1365-2567.2008.02827.x

[26] Damås, J.K., Smith, C., Øie, E., et al. (2007) Enhanced expression of the homeostatic chemokines CCL19 and CCL21 in clinical and experimental atherosclerosis: Possible pathogenic role in plaque destabilization. Arteriosclerosis, Thrombosis, and Vascular Biology, 27, 614-620. doi:10.1161/01.ATV.0000255581.38523.7c

[27] Trogan, E., Feig, J.E., Dogan, S., et al. Gene expression changes in foam cells and the role of chemokine receptor CCR7 during atherosclerosis regression in ApoE-deficient mice. Proceedings of the National Academy of Sciences of USA, 103, 3781-3786.

[28] Llodrá, J., Angeli, V., Liu, J., et al. (2004) Emigration of monocyte-derived cells from atherosclerotic lesions characterizes regressive, but not progressive, plaques. Proceedings of the National Academy of Sciences of USA, 101, 11779-11784.

[29] Adema, G.J. (2009) Dendritic cells from bench to bedside and back. Immunology Letters, 122, 128-130. doi:10.1016/j.imlet.2008.11.017 
[30] Pryshchep, O., Ma-Krupa, W., Younge, B.R., et al. (2008) Vessel-specific Toll-like receptor profiles in human medium and large arteries. Circulation, 118, 1276-1284. doi:10.1161/CIRCULATIONAHA.108.789172

[31] Ma-Krupa, W., Jeon, M. S., Spoerl, S., et al. (2004) Activation of arterial wall dendritic cells and breakdown of self-tolerance in giant cell arteritis. The Journal of Ex- perimental Medicine, 199, 173-183. doi:10.1084/jem.20030850

[32] Shiraki, R., Inoue, N., Kobayashi, S., et al. (2006) TollLike receptor 4 expressions on peripheral blood monocytes were enhanced in coronary artery disease even in patients with low C-reactive protein. Life Sciences, 80 , 59-66. doi:10.1016/j.lfs.2006.08.027 